\title{
Source performance metrics for EUV mask inspection
}

\author{
Larissa Juschkin $\odot$ and Daniel Wack \\ KLA Corporation, One Technology Drive, Milpitas, California, United States
}

\begin{abstract}
Rules are derived to obtain specifications on radiance, power, lifetime, and cleanliness of the source for an actinic patterned mask inspection system. We focus on the physical processes and technological aspects governing the requirements of radiation sources for reticle inspection. We discuss differences and similarities to scanner with respect to magnification, system etendue, and image recording. Source radiance requirements are estimated from a perspective of targeted throughput and defect detection sensitivity. The derivations consider the influence of photon shot noise on signal detection and conservation laws of light etendue and radiant flux. We describe the scaling laws for required radiance with targeted sensitivity index, optical contrast, field size, and system throughput. In addition, we address the limits on the required brightness and minimum repetition rate set by mask damage threshold. Finally, system and source cleanliness requirements and criticality of the source availability and lifetime are discussed. The analysis can be applied to other microscopy-based metrology and inspection applications. () The Authors. Published by SPIE under a Creative Commons Attribution 4.0 International License. Distribution or reproduction of this work in whole or in part requires full attribution of the original publication, including its DOI. [DOI: 10.1117/1.JMM.21.2.021204]
\end{abstract}

Keywords: extreme ultraviolet; mask inspection; mask; microscopy; etendue; laboratory sources; plasma sources; defect inspection; sensitivity; resolution; radiance; brightness.

Paper 21087SS received Nov. 17, 2021; accepted for publication Feb. 17, 2022; published online Mar. 8, 2022.

\section{Introduction}

At extreme ultraviolet (EUV) wavelengths, diffraction limited optical imaging (printing) with a lateral half-pitch resolution in the single-digit nanometer range is achievable with state-of-the-art reflective optics. ${ }^{1,2}$ The inspection of mask blanks and patterned masks (reticles) for EUV lithography at $13.5 \mathrm{~nm}$ requires the ability to scan large surfaces for the presence of small but printable defects as rapidly as possible. ${ }^{3}$ KLA is a global supplier of process control solutions for semiconductor manufacturing with more than 40 years of semiconductor process control experience. The first mask inspector was developed in 1978, and currently the RAPID division provides reticle inspection solutions for both mask shops and fabs. These solutions help ensure that lithography yields are consistently high, enabling cost-effective chip manufacturing. The ultimate goal is to find all critical defects on masks before these are printed in hundreds of thousands of chips. Another important feature is the ability of automated comparison of a photomask pattern with its database - designed pattern - to ensure the geometry is correct and errors can be avoided. Today, the relevance of both aspects only increases in importance.

Multiple inspections of a photomask occur through its lifecycle (see Fig. 1). In particular, mask substrates are inspected at the blank supplier. In the mask shop, patterned masks are inspected multiple times during fabrication, and during photolithography exposures in the wafer fab, masks are inspected periodically to ensure that the mask remains defect free. This is specifically critical for masks used in EUV lithography, for which these inspections are currently being performed at $193 \mathrm{~nm}$ wavelength in production. Two different illumination/imaging modes combined with advanced database modeling are used to enhance contrast and defect sensitivity. ${ }^{4}$ These tools support mask development and production down to $7 \mathrm{~nm}$ node.

However, as the structures shrink, despite being $4 \times$ larger at mask, one approaches limits of what is feasible with optical wavelengths. In defect inspection, similar as in microscopy, there is a triangle of trade-offs, and compromises are inevitable in defect inspection with respect to the

*Address all correspondence to Larissa Juschkin, larissa.juschkin@kla-tencor.com 

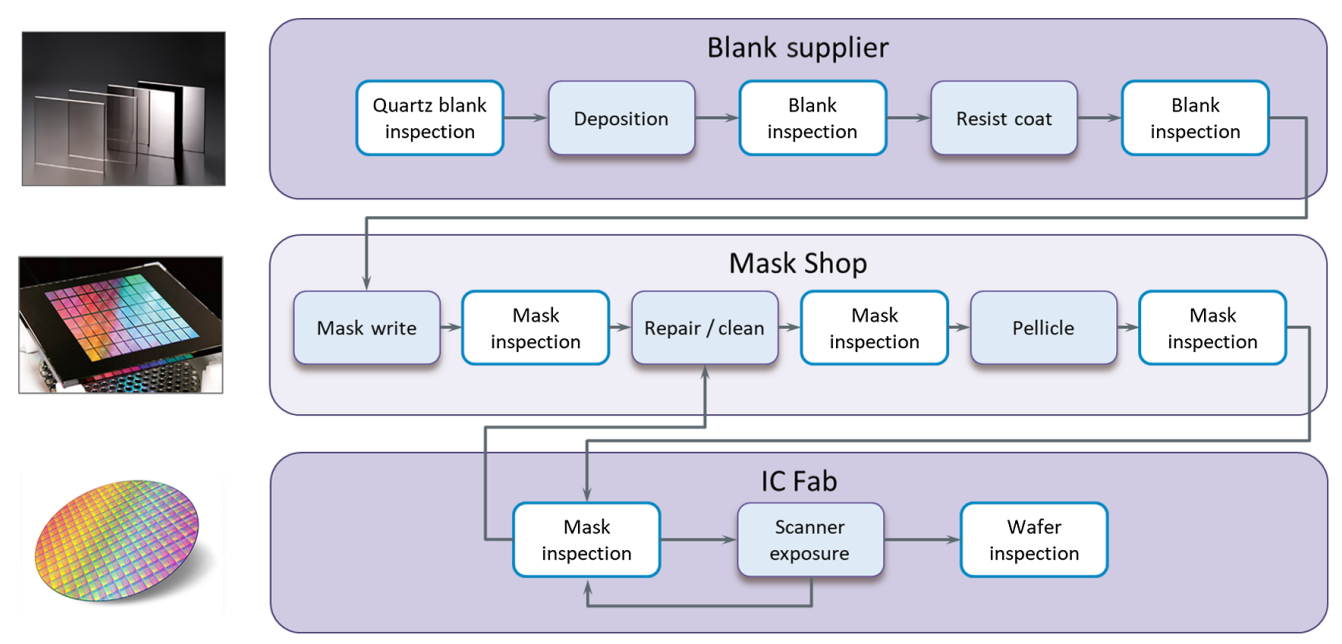

Fig. 1 EUV mask inspection flow: inspection is critical from blank supply through wafer fabrication.

throughput, resolution, and sensitivity. In the following, the requirements on source radiance are derived based on the desired throughput, optical resolution, and defect sensitivity, for the specific case of an actinic EUV mask inspector using basic illumination and imaging modes, and a single, simple defect type. Consideration of alternative and optimal illuminator and imaging modes is important, as works on optimizing EUV mask defect detection for specific defect types (e.g., phase defects) have shown. ${ }^{5-7}$ As our focus is on deriving source requirements as part of system design, for brevity we simplify the discussion by considering small, opaque defects under partially coherent illumination in a scanning imaging system. Thus, conclusions on optimal system designs to maximize actinic inspection performance and productivity, and the relative merits of actinic and electron beam inspection methods, will have to be discussed in subsequent work.

The structure of this paper is the following. After an introduction of the application of the patterned mask inspection and its image capture methodology, the scaling laws of required source brightness are derived. Then the minimum required source power is estimated for this application, and limits set by the mask damage threshold are reviewed. The final part underlines the requirements on source cleanliness and availability.

\section{Scanner versus Mask Inspector}

For such an EUV inspector, its optical architecture is going to look in many ways similar to that of an EUV scanner. There is a source-collector module, illuminator, mask, imaging optics, and detector (see Fig. 2 and Table 1). All optics, including mask itself, are reflective, mainly

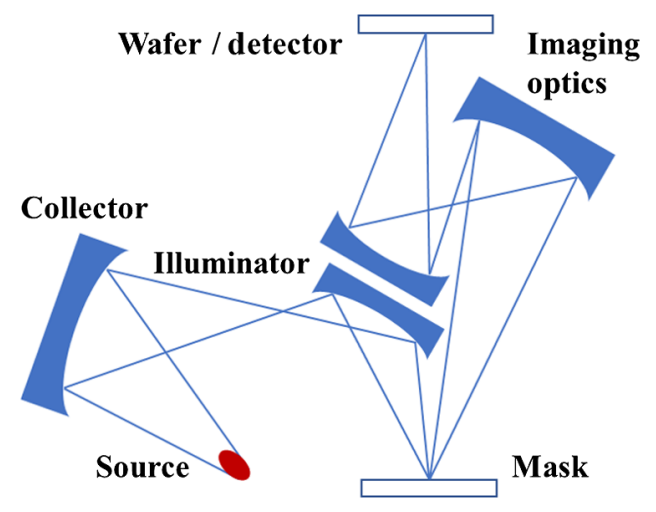

Fig. 2 Mask inspector: typical mask scan and image capture. Mask is moved across FOV during the image capture: photoinduced charge is shifted to the adjacent pixel columns synchronous with the motion of the image across the array of elements. 
Table 1 Differences and similarities between EUV scanner and mask inspector.

\begin{tabular}{lcc}
\hline \hline Parameter & Scanner & Inspector \\
\hline Source & High power & High brightness \\
Illuminator & Large etendue & Small etendue \\
Mask & Moving & Moving \\
Imaging optics & Demagnifying $(1 / 4 \times)$ large FOV & Magnifying $(500 \times$ to $1000 \times)$ small FOV \\
Wafer/detector & Moving & Static, charge transfer \\
Dose control & Pulse count modulation & Reference correction \\
\hline \hline
\end{tabular}

multilayer-based. The main difference will be in the imaging process. Instead of demagnifying mask structures onto a wafer, one projects a few hundred times magnified mask image onto a CCD-type detector. Because of such a large magnification, the field of view (FOV) and correspondingly the etendue (see Sec. 4) of an inspector are much smaller than that of a scanner. The recording medium is stationary, but photogenerated charge is transferred through the pixels synchronous with the mask motion. The dose control needs to ensure that all pixels are illuminated with a minimum number of photons and provide reference.

\section{Image Capture in Defect Inspection}

CCD-type detector is used in time delay integration (TDI) mode, which is well suited for lowlight applications and imaging of moving objects. ${ }^{8}$ The charge transfer through the detector pixels is synchronized with the object's image motion on detector such that every image pixel at mask, while moving through the illumination spot, contributes to the same bucket of photoinduced charge in the detector (see Fig. 3). Image moves on CCD at speed $v$; CCD line transfer is synchronized with transfer frequency $f$ :

$$
v=f \cdot p_{d}
$$

where $p_{d}$ is the detector pixel size. To increase the image acquisition rate, multiple readout channels/ports are used, which enable low-overhead imaging of high-speed objects. ${ }^{9}$

For a system with uniform illumination of the object field, the time for image capture $t$ of mask inspection area $A_{\text {mask }}$ (without overhead, i.e., after accounting for retrace (see Fig. 3), and ignoring partial image swaths at object edges), is determined by the pixel charge integration time $t_{\text {pix }}$ and detector pixel-array size:
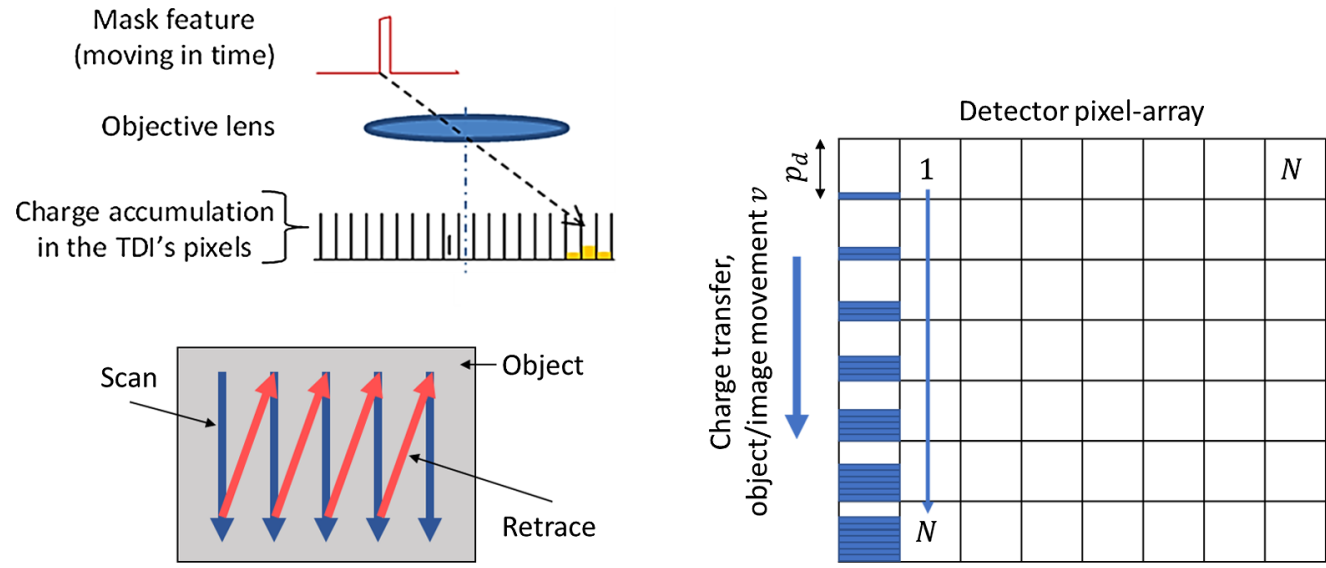

Fig. 3 Signal integration by TDI operation: integrating exposure of a moving object (inspired by technical documents available on Hamamatsu website ${ }^{8}$ ). 


$$
t_{\mathrm{pix}}=t \cdot \frac{\# \mathrm{pix}_{\mathrm{det}}}{\# \mathrm{pix}_{\mathrm{mask}}}=t \cdot \frac{N^{2} \cdot p^{2}}{A_{\mathrm{mask}}}
$$

where $N$ is detector pixel number in one direction, assuming a square detector, $p=p_{d} / M$ is pixel size at mask, $M$ is magnification of imaging system, \#pix mask $_{\text {is }}$ total number of inspection pixels at mask, and \#pix ${ }_{\mathrm{det}}=N^{2}$ is the number of detector pixels.

To minimize aliasing contributions to noise and ensure efficient use of the detector area, socalled critical sampling is usually applied, where pixel size at mask corresponds to half of the diffraction-limited resolution of the imaging system. This results in the point spread function sampling rate of 4.88:

$$
p=\frac{\lambda}{4 \cdot \mathrm{NA}_{\mathrm{im}}}, \quad a_{\mathrm{PSF}}=1.22 \cdot \frac{\lambda}{\mathrm{NA}_{\mathrm{im}}}, \quad \frac{a_{\mathrm{PSF}}}{p}=4.88,
$$

where $\lambda$ is the wavelength of light, $\mathrm{NA}_{\mathrm{im}}$ is numerical aperture of imaging optics, and $a_{\mathrm{PSF}}$ is diameter of the diffraction-limited point spread function (Airy disk).

The following equation summarizes different relations to calculate mask speed $v_{\text {mask }}$ :

$$
v_{\text {mask }}=\frac{p \cdot N}{t_{\text {pix }}}=\frac{A_{\text {mask }}}{t \cdot p \cdot N}=\frac{v}{M}=\frac{f \cdot p_{d}}{M}=f \cdot p .
$$

\section{Radiance, Etendue, and Resolution}

To obtain the required source in-band radiance for EUV mask inspection, the same approach is pursued as in previous works on high-resolution imaging. ${ }^{10,11}$ (In the following, all light metrics refer to in-band quantities, with roughly $2 \%$ spectral bandwidth determined by the integration of the product of source spectral distribution and the system spectral transmission function, largely driven by spectral reflectivity distribution for $\mathrm{Mo} / \mathrm{Si}$ multilayer coatings.) As EUV radiation is strongly absorbed by all materials, two simplifications are applied throughout the following considerations: First, the entire optical system is assumed to be in vacuum, and hence the refractive index of propagation medium is 1 and omitted in the formulas. Second, the effect of nonzero angle of incidence at mask is neglected. In reflection geometry, off-axis illumination is often required to separate incoming and outgoing light cones due to the lack of efficient beam splitters in EUV. However, the limited angular bandwidth of multilayer Bragg mirrors results in center ray angle of incidence at mask usually being smaller than $9 \mathrm{deg}$. For such illumination beams, the difference between the cross sectional and the actual illuminated areas is on the order of $1 \%$ or smaller which justifies the above approximation.

The radiance $L$ (also often called brightness) is the primary source characteristic which is, at best, conserved in an optical system. It is determined by radiant (photon) flux (or source power) divided by etendue. Etendue element $G$ is the phase space volume of a beam, given by a product of its cross section (area element projected in the direction of light propagation) and the solid angle subtended by the light beam (see Fig. 4 for definition of differential angle and area elements) ${ }^{10}$ :

$$
G=\int_{A} \int_{\Omega} \mathrm{d} A \cos \theta \cdot \mathrm{d} \Omega=\pi \cdot \mathrm{NA}^{2} \cdot A,
$$

where $A$ is an area element in the FOV, and NA $=\sin \alpha$ is the numerical aperture determined by the half-opening angle $\alpha$ to the next optical element. Etendue is conserved as light travels through free space and optical systems with perfect reflections or refractions which allows us to trace photons within each etendue element. The considered surface elementse.g., pixels — can be traced individually and independently through the imaging optical system between the detector and the sample. In the following, we will consider the etendue element determined by the resolved area at sample $A_{\text {to resolve }}$ and solid angle of illumination $\Omega_{\text {illumination }}$ (see Fig. 5). 


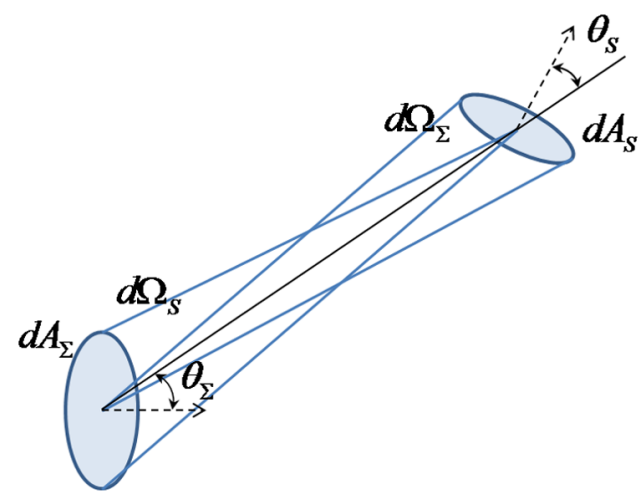

Fig. 4 Variables used in defining etendue and radiance: two differential surface elements, $\Sigma$ (source) and S (receiver), facing each other (adopted with permission from Ref. 10).

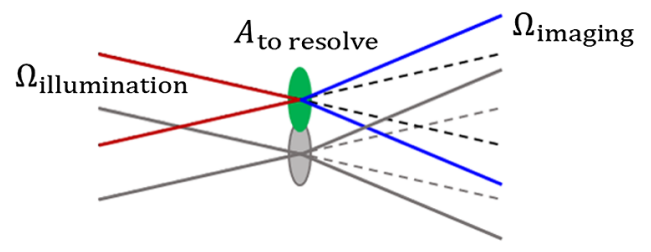

Fig. 5 Etendue elements at sample.

Photon flux is given by the number of photons $n_{\mathrm{ph}}$ hitting this area at sample divided by the pixel charge integration time $t_{\text {pix }}$. We must account for the photon energy $E_{\mathrm{ph}}$ to get power and divide it by the etendue to get the required brightness $L_{\text {sample }}$ at sample:

$$
L_{\text {sample }}=\frac{n_{\mathrm{ph}} \cdot E_{\mathrm{ph}} / t_{\mathrm{pix}}}{\Omega_{\text {illumination }} \cdot A_{\text {to resolve }}} .
$$

The radiance at sample can now be traced back to the source to obtain the source radiance $L_{\text {source }}$ after accounting for the losses in the system and wavelength dependency of photon energy $\left(E_{\mathrm{ph}}=h c / \lambda\right.$ with $h$ being Planck's constant and $c$ speed of light):

$$
L_{\text {source }}=\frac{n_{\mathrm{ph}} \cdot h c / \lambda}{T_{\text {sys }} \cdot t_{\text {pix }} \cdot \Omega_{\text {illumination }} \cdot A_{\text {to resolve }}},
$$

where all absorption losses are combined together and represented by the system transmission $T_{\text {sys }}$, including losses in illuminator (represented by transmission $T_{\text {ill }}$ ), imaging optics $\left(T_{\text {im }}\right)$, mask itself (with pellicle, $T_{\text {mask }}$ ), and detector (quantum efficiency, QE):

$$
T_{\text {sys }}=T_{\text {ill }} \cdot T_{\text {mask }} \cdot T_{\text {im }} \cdot \mathrm{QE} .
$$

To calculate the minimum resolved area element, assuming diffraction limited system with partially coherent illumination, one must account for both imaging and illumination NA, based on Abbe's criterion. ${ }^{12,13}$ The ratio of sample-side numerical apertures of illumination $\left(\mathrm{NA}_{\mathrm{ill}}\right)$ and imaging $\left(\mathrm{NA}_{\mathrm{im}}\right)$ systems is usually denoted as $\sigma$. In the product of the resolved (square) area element and illumination solid angle (defining the considered etendue element), the NA is therefore cancelled, and only $\sigma$ is left as optical parameter:

$$
\begin{gathered}
A_{\text {to resolve }}=\left(\frac{\lambda}{\mathrm{NA}_{\mathrm{im}}+\mathrm{NA}_{\mathrm{ill}}}\right)^{2}, \quad \sigma=\frac{\mathrm{NA}_{\mathrm{ill}}}{\mathrm{NA}_{\mathrm{im}}}, \\
\Omega_{\text {illumination }}=\pi \cdot \mathrm{NA}_{\mathrm{ill}}^{2}=\pi \cdot \sigma^{2} \cdot \mathrm{NA}_{\mathrm{im}}^{2},
\end{gathered}
$$




$$
\Omega_{\text {illumination }} \cdot A_{\text {to resolve }}=\pi \cdot \lambda^{2} \cdot \frac{\sigma^{2}}{(1+\sigma)^{2}} .
$$

Combining all together, with the image capture time derived in previous Sec. 3, we get the following dependency for the required radiance:

$$
L_{\text {source }}=\frac{h c}{\pi \lambda^{3}} \cdot \frac{n_{\mathrm{ph}} \cdot A_{\mathrm{mask}}}{T_{\mathrm{sys}} \cdot t \cdot N^{2} \cdot p^{2}} \cdot \frac{(1+\sigma)^{2}}{\sigma^{2}} .
$$

Now, we calculate how many photons are needed to hit the resolution area.

\section{Contrast and Sensitivity Index}

Let us consider two small neighboring area elements, one containing a distinct feature and another one representing its surrounding (background) (see Fig. 6). When using light to obtain an image, differentiating the feature from the background means detecting a difference in the photon numbers (signals) from the two area elements. We know that counting photons follows Poisson statistics. It means, e.g., if we consider 2500 photons detected from a transparent background "pixel," if repeated the detector response is not going to always be 2500 but will follow the probability distribution with the spread $\delta_{\text {ph }}$ given by the square root of the number of photons $\delta_{\mathrm{ph}}=\sqrt{n_{\mathrm{ph}}}$ (see Fig. 7). The signal from the neighboring pixel, of $90 \%$ transmission in this

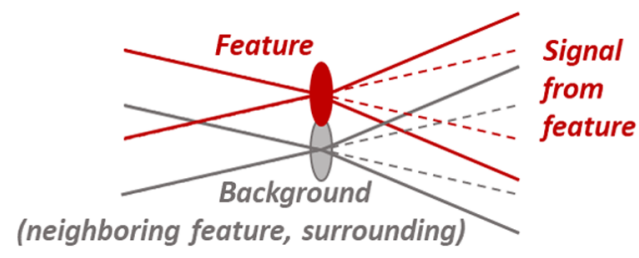

Fig. 6 Light signals captured by imaging optics from different illuminated structures at sample. For absorbing features on more transparent background, signals from features are smaller.

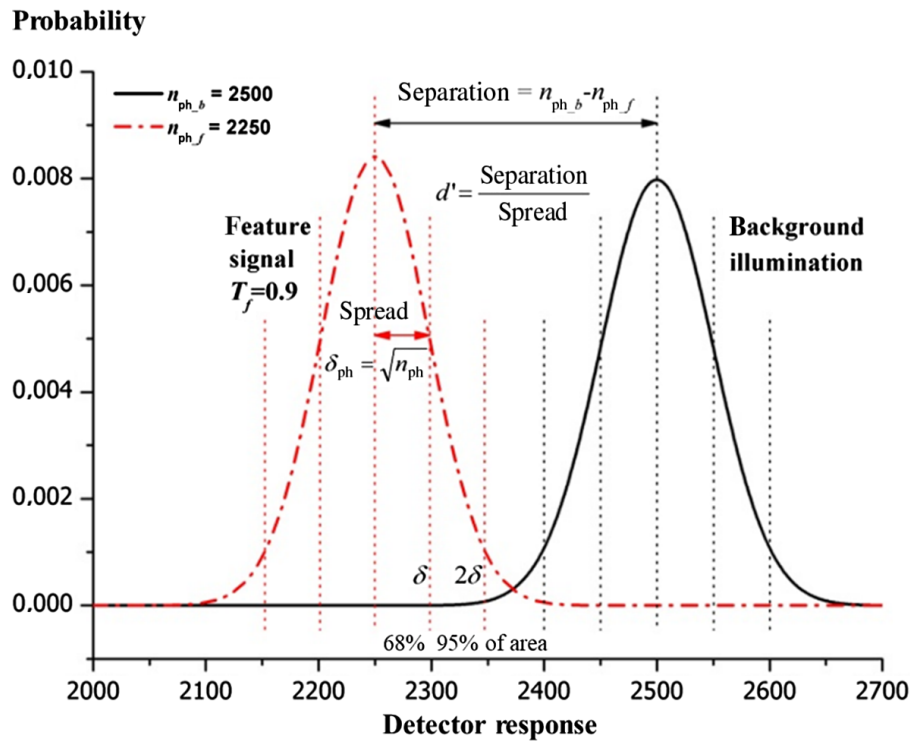

Fig. 7 Response probabilities of occurrence for two photon signals on an ideal detector: the right black solid line corresponds to the background illumination, the left red dash-dot line to an object feature of $10 \%$ absorption. The illumination level of 2500 photons is adjusted to just satisfy the requirement on separation to noise ratio of $d^{\prime} \cong 5$ for $\sim 100 \%$ certainty in distinguishing between both signals (adopted with permission from Ref. 10). 
Defect image

(die)

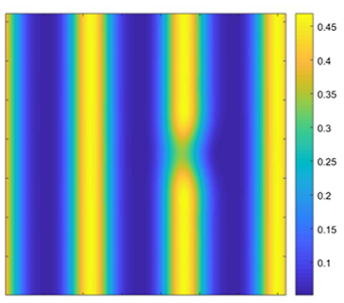

Ideal image (reference or database)

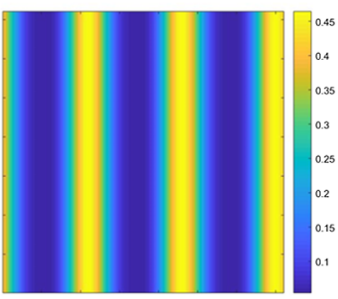

Defect signal

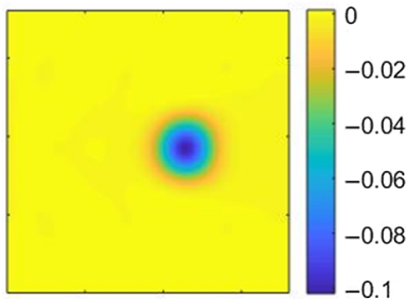

Fig. 8 Schematic diagram of defect signal capture in patterned mask inspection (inspired by Refs. 15 and 16).

example, will also follow the Poisson statistics. To be sure that the detector response corresponds to the physical feature, the separation (difference) of signal from background must be large enough compared to the statistical spread. The Rose criterion in microscopy states that signal difference to noise ratio must be larger than 5 for $\sim 100 \%$ certainty in distinguishing image features. If we consider $5 \delta$ separation as in this example, one can see that $98 \%$ of the area lies within $\pm 2.5 \delta$, which explains Rose criterion obtained experimentally. ${ }^{14}$

In microscopy, contrast is defined as a ratio of difference between two signals to their sum and is used as a measure of image quality. In many cases, it can be approximated as a ratio of signal difference to signal maximum. Signal (photon) noise is given by the square root of sum of two signals or approximately the square root of signal maximum because of the summation in quadrature. The consideration of these relations and Rose criterion allows one to derive the minimum number of photons for background illumination in microscopy. ${ }^{10,11}$

For the mask inspection application, this consideration needs to be modified slightly as we are not comparing the signals from the two neighboring pixels but the signals from the same pixel in two different images. One image is obtained experimentally and may contain a defect, another image is a reference, without defect, either from an experiment, or from modeling (see Fig. 8). ${ }^{15,16}$ The image itself is pattern-dependent, therefore, the convention for normalization is to use a signal from blank mask multilayer, which results in the following definition of contrast:

$$
C=\frac{n_{\text {with defect }}-n_{\text {without defect }}}{n_{\text {blank multilayer }}}=\frac{\Delta n}{n} .
$$

Here $n$ denotes the number of photons detected from a resolution area element $A_{\text {to resolve }}$ on a blank mask multilayer, and $\Delta n$ is the difference in photon numbers from area elements of the same size on a patterned mask with a defect and a defect-free reference pattern. That is, we are considering two signals, one from a (background) pattern and another one from a pattern with a defect and relate them to the blank multilayer signal. The probability distribution gets slightly modified: after image subtraction, one can get also negative signal and noise (see Fig. 9). In addition to that, signal spread $\delta$ may be also different as there are other sources of noise on

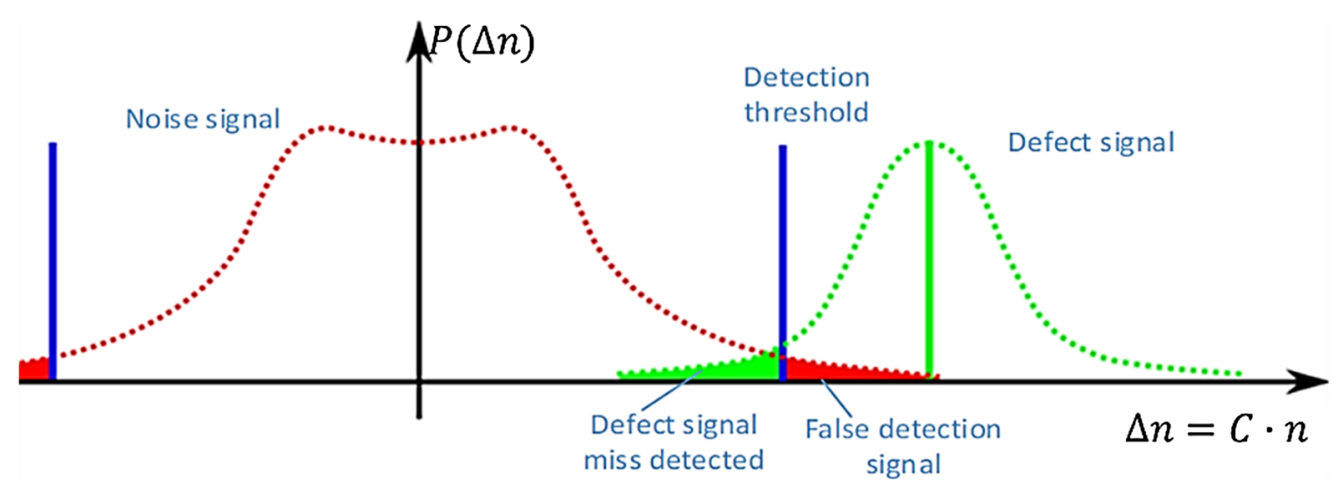

Fig. 9 Probability distribution of defect and noise signals in patterned mask inspection. 
top of the photon signal (shot) noise. To account for that, let us introduce a noise overhead $\varepsilon$ with respect to shot noise:

$$
\delta=(1+\varepsilon) \cdot \delta_{\mathrm{ph}} \approx(1+\varepsilon) \cdot \sqrt{n} .
$$

Usually, a measurement system is optimized to be signal noise dominated, i.e., $\varepsilon$ can be considered small.

The area under the noise distribution protruding into the signal corresponds to potential false defect detection and that of a signal within the noise range to potential missed defects. So, to discriminate defect signals from false events, one selects a threshold based on the targeted defect capture rate and balanced against false event rate (see Fig. 9).

With all the information above, we can now answer the question of how many photons are needed to be detected from a blank multilayer pixel to ensure the targeted defect capture rate. The ratio of separation to spread is called sensitivity index $d^{\prime},{ }^{10}$ and it needs to be larger than a selected threshold TNR:

$$
d^{\prime}=\frac{\Delta n}{\delta}=\frac{C \cdot n}{(1+\varepsilon) \cdot \sqrt{n}} \geq \mathrm{TNR}
$$

where TNR is the threshold-to-noise ratio, as opposite to SNR, signal-to-noise ratio, which is a characteristic of a (difference) image. TNR is a number determining the smallest usable value of SNR. Combined altogether, one gets the following requirement on the photon number:

$$
n \geq\left(\frac{(1+\varepsilon) \cdot \mathrm{TNR}}{C}\right)^{2} \approx \frac{\mathrm{TNR}^{2}}{C^{2}} .
$$

Now the next question is how to estimate the contrast. When optimizing system performance, defect sizes of interest are subresolution; depending on the analysis goals, to estimate image contrast with adequate accuracy, a rigorous diffraction calculation may be necessary. In the preliminary stage of system analysis and the simplest case of an absorbing defect, one can roughly estimate the contrast based on the transmission of the area covered by the defect and that of the resolved area, i.e., it is proportional to the ratio of the defect area to the resolved area:

$$
C \cong \frac{\left(T_{\text {defect }}-T_{\text {pattern }}\right) \cdot A_{\text {defect }}}{T_{\text {mask blank }} \cdot A_{\text {to resolve }}}
$$

For a small (absorbing) defect on a multilayer, it is just the ratio of areas:

$$
C \approx \frac{-A_{\text {defect }}}{A_{\text {to resolve }}}
$$

We are considering a case of subresolution detection here, i.e., with defect area being smaller than the resolved area.

In the general case, including scattering defects, the contrast is defect and system-dependent and is determined by the corresponding scattering cross sections of pattern structures on mask, of pattern structures with a defect and of blank multilayer, and objective NA (solid angle). It is further influenced by illumination conditions. In Fig. 10, one can see a typical bright field microcopy path, with imaging NA larger than illumination NA to increase relative weight of scattered light. In dark-field microscopy, only scattered light is collected. In phase contrast microscopy, the directly transmitted light is modified to increase contrast. All these techniques may be applied to optimize a metrology/inspection system for higher contrast (signal) during defect detection. We refer to the study performed at UC Berkeley to explore all these options and the expected SNR in an inspection system as a function of different parameters. ${ }^{15-17}$ As an example, the influence of multilayer scatter noise needs to be considered as well, i.e., the best focus is defined by the minimum speckle contrast from mask roughness. Pixel size, defocus, $\sigma$, and photon count per pixel will all influence the resulting SNR. 


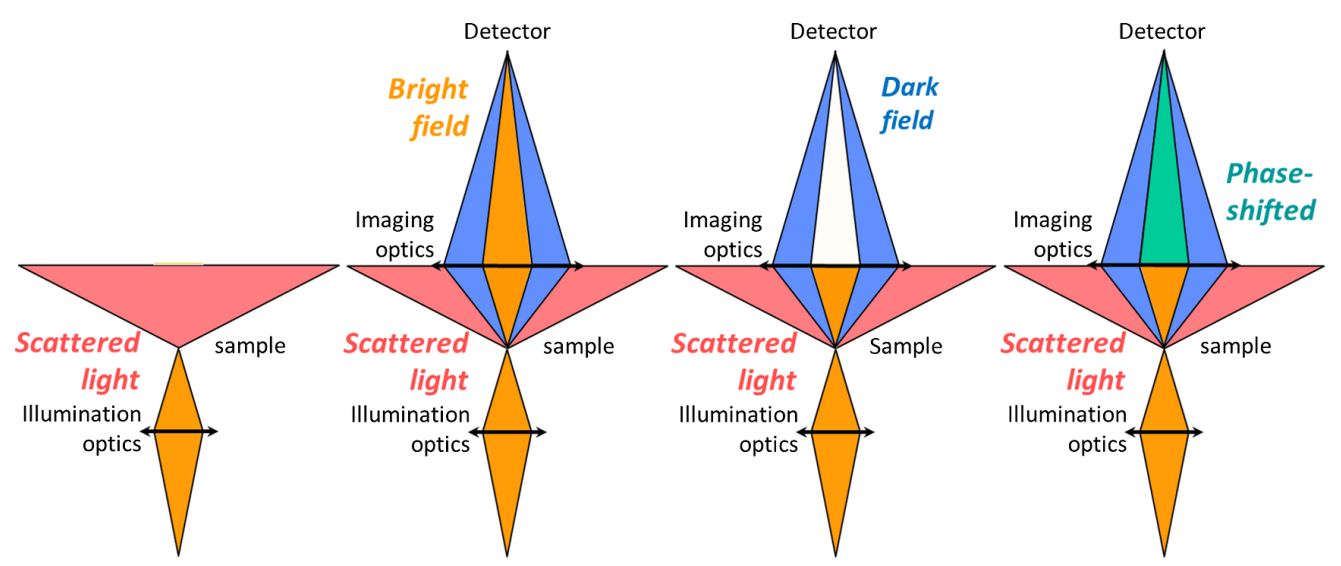

Fig. 10 Image capture and contrast enhancing techniques in microscopy.

\section{Defect Printability and Critical Defects}

To get an estimate of the required number of photons, let us now consider the goal of mask inspection. One needs to find those defects which will print during lithography process. In lithography, the defect impact is measured by the error in structure size it introduces, its $\mathrm{CD}$ change (CD: critical dimension and $\triangle \mathrm{CD}$ : $\mathrm{CD}$ error). The specification on minimum photon flux is therefore governed by defects which are most challenging to detect. Performed studies, as the one by Kamo et al., ${ }^{18}$ indicate that $\Delta \mathrm{CD} / \mathrm{CD}$ scales roughly proportional to the defect area (see Fig. 11). Recognizing that the inspection goal is to detect all defects at a given value of printing error (e.g., $10 \% \Delta \mathrm{CD} / \mathrm{CD}$ ), one can see that the most challenging defects (i.e., the same change in $\mathrm{CD}$ is coming from a smaller defect) are of protrusion type: an absorber extended to the multilayer region. For protrusion defects, we can use the scaling from Fig. 11 and get that $\Delta \mathrm{CD} / \mathrm{CD}$ is given by the squared ratio of defect size to the half pitch at mask hp (assuming that pattern is resolved and taking into account that structures at mask are $4 \times$ larger than on wafer):
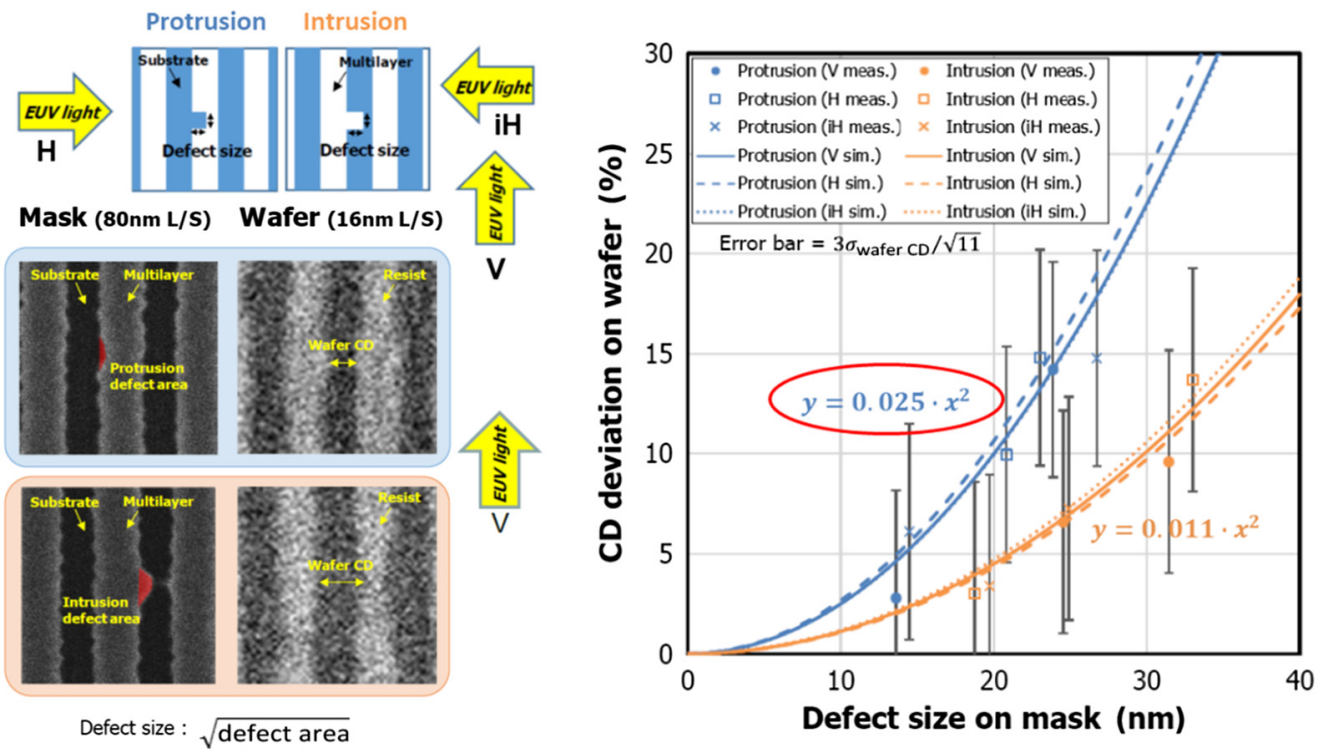

Fig. 11 Defect printability study by Kamo et al.: ${ }^{18}$ impact of mask defects is measured by the introduced $C D$ change on wafer. The most challenging (i.e., with larger impact from smaller defects) are defects of protrusion type which are essentially small absorbers on multilayer (data analyzed and adopted with permission from Ref. 18). 


$$
\frac{\Delta \mathrm{CD}}{\mathrm{CD}} \approx \frac{A_{\text {defect }\left(\mathrm{nm} m^{2}\right)}}{4000} \cong\left(\frac{a_{(\mathrm{nm})}}{4 \cdot 16}\right)^{2}=\left(\frac{a}{\mathrm{hp}}\right)^{2},
$$

where $a=\sqrt{A_{\text {defect }}}$ is the effective defect size, and the investigated half pitch at wafer is $16 \mathrm{~nm}$ (see Fig. 11).

On the other hand, a protrusion defect corresponds to an absorber on a multilayer, exactly the case considered earlier, where the contrast in an inspection tool is given by the ratio of defect area to the resolved area. Given this, we can use targeted $\triangle \mathrm{CD} / \mathrm{CD}$ to estimate the corresponding defect contrast:

$$
|C| \approx \frac{A_{\text {defect }}}{A_{\text {to resolve }}} \approx\left(\frac{a}{h p}\right)^{2} \cong \frac{\Delta \mathrm{CD}}{\mathrm{CD}} .
$$

As an example, with TNR of 7 and noise overhead of $0.1,6000$ detectable photons are needed within hp-defined area to detect defects resulting in $10 \% \mathrm{CD}$ change.

\section{Scaling Laws for Required Source Radiance and Power}

Combining all together, we obtain the following scaling laws of the required in-band source brightness for actinic patterned mask inspection (APMI) application:

$$
L_{\text {source }}=\frac{\mathrm{hc}}{\pi \lambda^{3}} \cdot\left(\frac{(1+\varepsilon) \cdot \mathrm{TNR}}{C}\right)^{2} \cdot \frac{(1+\sigma)^{2}}{p^{2} \cdot \sigma^{2}} \cdot \frac{A_{\mathrm{mask}}}{T_{\mathrm{sys}} \cdot t \cdot N^{2}} .
$$

The first fraction-multiplier term in this expression combines the constants and shows wavelength dependency: $1 / \lambda^{2}$ comes from the resolved area (in an etendue element) and $1 / \lambda$ from photon energy, thus overall $1 / \lambda^{3}$ scaling. The second term is determined by the defect sensitivity with $C$ being optical contrast of a critical defect. The third fraction-multiplier term represents the optical system influence on contrast and resolution. Here $p$ is the pixel size at mask. The last term is determined by the targeted throughput.

For a practical estimate, we can use the targeted defect sensitivity in terms of $\triangle \mathrm{CD} / \mathrm{CD}$ and the half pitch at wafer $\mathrm{hp}_{\text {wafer }}$ and assume that mask half pitch is resolved in an inspection tool, and optical contrast is comparable to that of a scanner for printable defects. This results in the following dependency:

$$
L_{\text {source }}=\frac{h c}{\pi \lambda^{3}} \cdot\left(\frac{(1+\varepsilon) \cdot \mathrm{TNR}}{\Delta \mathrm{CD} / \mathrm{CD}}\right)^{2} \cdot \frac{A_{\mathrm{mask}}}{\mathrm{hp}_{\mathrm{wafer}}^{2} \cdot \sigma^{2} \cdot T_{\mathrm{sys}} \cdot t \cdot N^{2}},
$$

which is also illustrated in Fig. 12(a) for a stated set of realistic conditions, e.g., system transmission on the order of $1 \%$ (assuming grazing incidence optics reflectivity, pellicle transmission and detector $\mathrm{QE}$ of $\sim 80 \%$, and seven multilayer mirror reflections of $\sim 60 \%$ ). One can see that minimum required source radiance lies in the range of about 10 to $100 \mathrm{~W} /\left(\mathrm{mm}^{2} \mathrm{sr}\right)$ depending on the detector pixel number.

Let us now consider the source power needed for mask inspection. One can get it from the radiance by multiplying it with the detection etendue which is given by the total number of detector(s) pixels times pixel etendue, $G_{\text {pix }}=\pi \cdot \lambda^{2} \cdot \sigma^{2} / 16$. One has also to account for power losses due to overfill in the illumination FOV and solid angle: etendue fill factor $F$. The resulting dependency is summarized in the following expression:

$$
P_{\text {source }}=L_{\text {source }} \cdot \frac{N^{2} \cdot G_{\mathrm{pix}}}{F}=\frac{\mathrm{hc}}{16 \lambda} \cdot\left(\frac{(1+\varepsilon) \cdot \mathrm{TNR}}{C}\right)^{2} \cdot \frac{A_{\mathrm{mask}} \cdot(1+\sigma)^{2}}{F \cdot T_{\mathrm{sys}} \cdot t \cdot p^{2}} .
$$

Using the estimate for the contrast obtained above (in terms of $\Delta \mathrm{CD} / \mathrm{CD}$ ) and half pitch at wafer, we get the following dependency of the source power [see also an illustration in Fig. 12(b)]: 


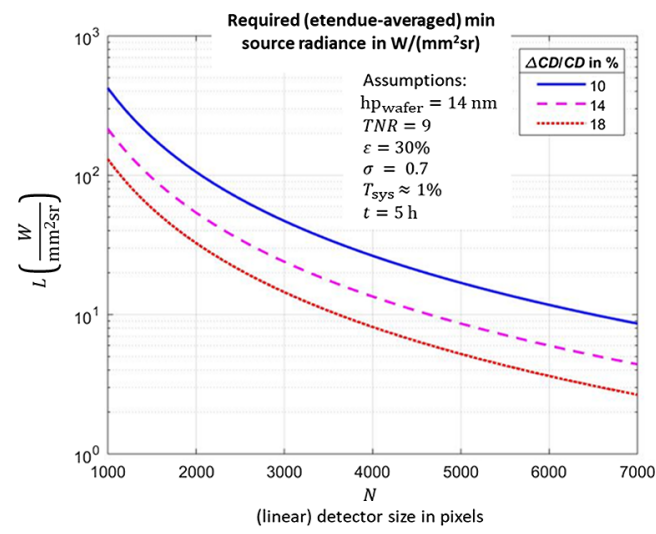

(a)

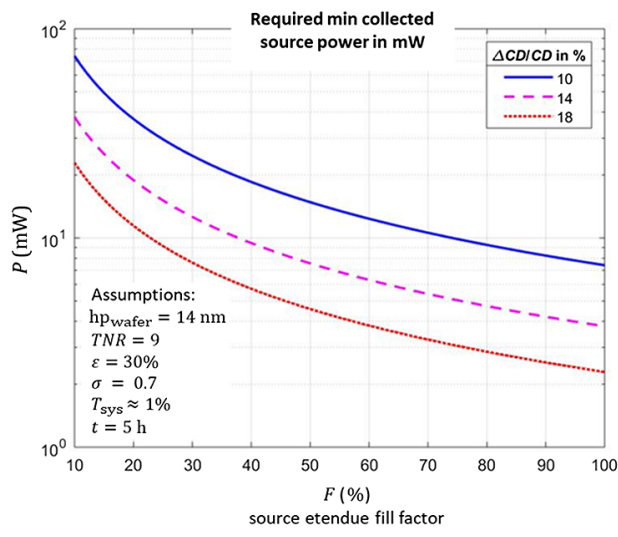

(b)

Fig. 12 (a) Scaling laws of the required in-band source radiance as a function of linear detector size and (b) in-band source power as a function of etendue fill factor for an EUV mask inspector with a set of assumed parameters summarized in the graphs.

$$
P_{\text {source }}=\frac{\mathrm{hc}}{16 \lambda} \cdot\left(\frac{(1+\varepsilon) \cdot \mathrm{TNR}}{\Delta \mathrm{CD} / \mathrm{CD}}\right)^{2} \cdot \frac{A_{\mathrm{mask}}}{F \cdot T_{\mathrm{sys}} \cdot t \cdot \mathrm{hp}_{\mathrm{wafer}}^{2}} .
$$

The required minimum collected source power is independent of the detector pixel number. It is determined by the required photon count per pixel, throughput, and the total number of pixels at mask. More than 20 terapixels need to be inspected with at least 1000 detected photons per pixel, which results in the source power ranging from a few $\mathrm{mW}$ to almost $100 \mathrm{~mW}$ depending on other parameters. As an example, this is beyond of what is achievable with high harmonic generation (HHG) EUV sources. For mask metrology, where a limited number of defects need to be reviewed, HHG EUV sources might be considered because of their high brightness, but for inspection of the entire mask, their power is not sufficient. ${ }^{19-21}$

\section{Mask Damage}

Another aspect which must be considered is the risk of mask damage during inspection. To calculate irradiance at mask, we need to multiply radiance at mask with solid angle of illumination. Here is the resulting relation where system transmission is replaced by that of imaging optics, mask (with pellicle), and detector:

$$
E_{\mathrm{mask}}=L_{\mathrm{mask}} \cdot \Omega_{\mathrm{ill}}=\frac{\mathrm{hc}}{16 \lambda} \cdot\left(\frac{(1+\varepsilon) \cdot \mathrm{TNR}}{C}\right)^{2} \cdot \frac{A_{\mathrm{mask}} \cdot(1+\sigma)^{2}}{T_{\mathrm{mask}} \cdot T_{\mathrm{im}} \cdot \mathrm{QE} \cdot t \cdot N^{2} \cdot p^{4}} .
$$

The example plotted in Fig. 13(a) is obtained again using $\triangle \mathrm{CD} / \mathrm{CD}$ and half pitch at wafer for contrast/sensitivity estimate:

$$
E_{\text {mask }}=\frac{\mathrm{hc}}{16 \lambda} \cdot\left(\frac{(1+\varepsilon) \cdot \mathrm{TNR}}{\Delta \mathrm{CD} / \mathrm{CD}}\right)^{2} \cdot \frac{A_{\text {mask }}}{T_{\mathrm{mask}} \cdot T_{\mathrm{im}} \cdot \mathrm{QE} \cdot t \cdot N^{2} \cdot \mathrm{hp}_{\mathrm{wafer}}^{4} \cdot(1+\sigma)^{2}} .
$$

Regarding damage threshold, the respective studies $^{22,23}$ show that fluence per pulse must be considered which is dependent on irradiance at mask and source frequency. The onset of damage mechanisms for patterned mask is observed at fluences per pulse as low as $10 \mathrm{~mJ} / \mathrm{cm}^{2} .^{22}$ Damage mechanisms are mainly thermally activated and thermomechanical processes. ${ }^{23}$ The interdiffusion of Mo and $\mathrm{Si}$ leads to formation of $\mathrm{MoSi}_{2}$ which is a denser material, which results in compaction. ${ }^{24}$ The multilayer period is consequently reduced and thus reflectivity changes. The challenging requirement for mask inspection is that reflectivity changes stay below $0.1 \%$ for 25 inspections, which means that changes in lattice constant should be smaller than $5 \mathrm{pm}$. 


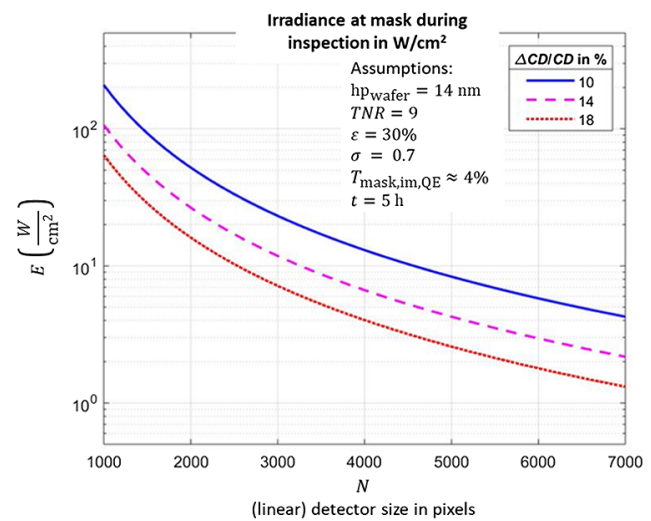

(a)

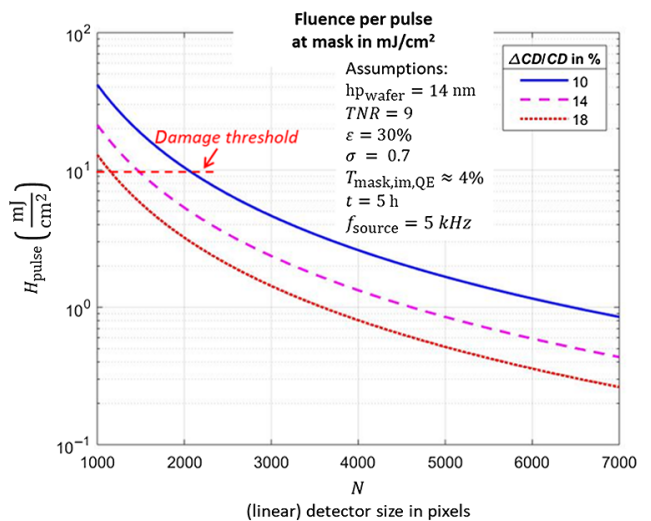

(b)

Fig. 13 (a) Scaling laws of the irradiance at mask and (b) fluence per pulse as a function of linear detector size for an EUV mask inspector with a set of assumed parameters summarized in the graphs.

Figure 13(b) shows the corresponding scaling of fluence per pulse assuming source repetition rate of $5 \mathrm{kHz}$. One can see that one can actually get into the regime where mask damage may occur. References 22-26 provide insights into different processes which may cause damage or modification of mask multilayer. For example, in addition to compaction, surface roughening may occur caused by accumulation of plastic deformation under cyclic thermoelastic strain induced by pulsed EUV illumination. If absorbed energy heats the multilayer above $325^{\circ} \mathrm{C}$, the $\mathrm{MoSi}_{2}$ layer crystallizes, followed by complete intermixing causing significant reduction of reflectivity.

For parameters that result in fluence per pulse equal to damage threshold, we can trace back what is the corresponding source brightness and find $100 \mathrm{~W} /\left(\mathrm{mm}^{2} \mathrm{sr}\right)$ for $5 \mathrm{kHz}$ repetition rate [see Fig. 13(b)]. This sets upper limit on source brightness (at certain frequency) or (ultimately) the lower limit on its repetition rate, and when combined with the results in Fig. 12, indicates that for the highest performance EUV mask inspection, the APMI system design is likely to face constraints, for example on minimum detector size.

In addition to mask damage, one must also consider risks of adding particle contamination defects. EUV mask inspectors are the cleanest machines among all tools used in a fab or mask shop since they are supposed to find defects and not introduce any. Ultraclean vacuum and particle control are extremely critical for mask environment. As an example, current spec for Teron $640 \mathrm{e}^{4}$ is $<0.1$ particles larger than $30 \mathrm{~nm}$ added per reticle pass (and 0 above $120 \mathrm{~nm}$ ). This requirement is going to tighten in the future as the structures shrink.

\section{Source Debris and Life Time}

Although plasma-based sources are efficient EUV emitters, these are known to be the dirtiest subsystems in EUV tools. The problem of isolating the mask from source debris is more severe in an inspector than in a scanner. Based on its optical constants, tin is a strong absorber, with the real part of refractive index close to that of a Ta-based absorber, but the extinction coefficient $k$ twice higher. For a tin-based source, tin reaching the mask generates therefore critical (absorber) defects. The best values targeted and achieved in scanners are one adder per 10,000 wafers. ${ }^{27}$ At 140 wafers/h, this corresponds to $\sim 70 \mathrm{~h}$ of exposures. 2016 status was 120 adders. ${ }^{27}$ Assuming $5 \mathrm{~h}$ per mask inspection, these numbers can be translated to at least one adder per $\sim 15$ inspections! 2016-equivalent data would correspond to about 10 adders per one inspection! These values are not acceptable.

There are several mechanisms how tin may reach the mask. This happens mainly due to secondary effects of bounced/released microparticles, liquid tin splashing, and accumulation (and subsequent sputtering) of deposited tin at the walls. ${ }^{28,29}$ A thin-film membrane separating the source from the rest of the system could improve the protection but introduces its own issues 
and particulation risks if ruptured. An EUV source for reticle inspection must comply with the tight cleanliness requirements.

Closely related to particles debris from source is its lifetime. In plasma-based sources, to produce EUV light, matter is ablated more than 5000 times per s. Solving the problem of target recovery and lifetime is therefore the biggest challenge. This includes lifetime of any component facing plasma or near it. Let us consider an example of electrode erosion in discharges, where published data is available. Electrode mass loss is about $1 \mathrm{~g}$ per mega-shot ${ }^{30}$ which corresponds to $5 \mathrm{~kg}$ per 10 days. Even for recovering liquid-tin coated electrodes, it is a huge consumption. Electrode lifetime of 2 billion shots is reported for LDP concept ${ }^{31}$ which translates to about 5 days at $5 \mathrm{kHz}$. For an inspection tool, replacement of components every week is not an option. Maintenance time of 5\% to $10 \%$ represents a serious problem for the inspection system availability. The source must be highly reliable to avoid impact to overall system availability. In patterned mask inspection, every $\%$ of tool availability is so critical that anything $<85 \%$ is unlikely to gain adoption by mask shops that run 24/7. Inspection system providers are evaluating or developing plasma source technologies which can contain Sn debris or avoid it altogether. ${ }^{32,33}$

\section{Conclusions}

We presented the guidelines to determine requirements on inspection source brightness. The required radiance may vary significantly depending on technical realization of an inspection tool. Minimum needed source radiance of about 10 to $50 \mathrm{~W} /\left(\mathrm{mm}^{2} \mathrm{sr}\right)$ scales quadratically with the targeted defect sensitivity and inversely with throughput and detector pixel number. Minimum needed source power is estimated to be about 1 to $100 \mathrm{~mW}$. It is determined by photon count per pixel, pixel number at mask and throughput and is independent of detector pixel number. Mask damage threshold sets upper limit on source brightness and lower limit on its repetition rate, e.g., $\sim 100 \mathrm{~W} /\left(\mathrm{mm}^{2} \mathrm{sr}\right)$ at $5 \mathrm{kHz}$. Higher repetition rates are favorable. These inspectors are the cleanest machines among all tools used in a fab/mask shop. Contamination and particle control are extremely critical. The source subsystem must have high reliability to avoid impact to the overall system availability. Every $1 \%$ availability loss is critical for mask shops that run 24/7. Weekly maintenance is not an option.

\section{Acknowledgments}

L. J. appreciates the exciting and helpful discussions with Fergal O'Reilly from the University College Dublin, Klaus Bergmann and Serhiy Danylyuk from Fraunhofer Institute for Laser Technology in Aachen, and Konstantin Tsigutkin from KLA Corporation at different stages of gaining insights into the complex interrelations of high-resolution imaging applications with laboratory EUV sources.

\section{References}

1. T. Brunner et al., "EUV dark field lithography: extreme resolution by blocking 0th order," Proc. SPIE 11609, 1160906 (2021).

2. J. van Schoot et al., "High-numerical aperture extreme ultraviolet scanner for 8-nm lithography and beyond," J. Micro/Nanolithogr. MEMS MOEMS 16(4), 041010 (2017).

3. V. Bakshi Ed, EUV Lithography, SPIE Press, Bellingham, Washington (2018).

4. W. Broadbent et al., "1X HP EUV reticle inspection with a $193 \mathrm{~nm}$ inspection system," Proc. SPIE 10451, 104510M (2017).

5. T. Yamane et al., "The performance of an actinic full-field EUVL mask blank inspection system," Proc. SPIE 7271, 72713H (2009).

6. T. Terasawa et al., "Actinic mask blank inspection and signal analysis for detecting phase defects down to $1.5 \mathrm{~nm}$ in height," Jpn. J. Appl. Phys. 48, 06FA04 (2009).

7. T. Terasawa et al., "Actinic phase defect detection and printability analysis for patterned EUVL mask," Proc. SPIE 7636, 763602 (2010). 
8. Technical information, "Back-thinned TDI-CCD," Cat. No KMPD9004E03, Hamamatsu Photonics K.K., Solid State Division, Hamamatsu City, Japan, 2015, https://www .hamamatsu.com/resources/pdf/ssd/tdi-ccd_kmpd9004e.pdf.

9. D. L. Brown and Y.-H. Chuang, "Continuous clocking of TDI sensors," United States Patent US 2006/0103725 A1 (2006).

10. L. Juschkin, "Imaging with plasma based extreme ultraviolet sources," Proc. SPIE 8678, 86780F (2012).

11. L. Juschkin, "Source radiance requirements for high-resolution imaging and interference techniques," in Proc. 2014 Int. Workshop EUV and Soft X-Ray Sour., Dublin, http:// www.euvlitho.com/2014/2014\%20Source\%20Workshop\%20Proceedings.pdf (2014).

12. E. Abbe, "Beiträge zur Theorie des Mikroskops und der mikroskopischen Wahrnehmung," Archiv f. mikrosk. Anatomie 9, 413-468 (1873).

13. M. Born and E. Wolf, "The diffraction theory of aberrations," in Principles of Optics: Electromagnetic Theory of Propagation, Interference and Diffraction of Light, pp. 517553, Cambridge University Press, Cambridge (1999).

14. A. Rose, "Television pickup tubes and the problem of vision," in Advances in Electronics, L. Marton, Ed., Vol. 1, pp. 131-166, Academic Press, New York (1948).

15. Y.-G. Wang, "Key challenges in EUV mask technology: actinic mask inspection and mask 3D effects," PhD thesis, UC Berkeley (2017).

16. Y.-G. Wang et al., "Phase-enhanced defect sensitivity for EUV mask inspection," Proc. SPIE 9235, 92350L (2014).

17. Y.-G. Wang, A. Neureuther, and P. Naulleau, "Impact of noise sources and optical design on defect detection sensitivity in extreme ultraviolet actinic pattern inspection tool," J. Micro/ Nanolithogr. MEMS MOEMS 16(1), 013504 (2017).

18. T. Kamo et al., "Mask 3D effect reduction and defect printability of etched multilayer EUV mask," Proc. SPIE 10957, 109571C (2019).

19. D. G. Lee, "Actinic tools using coherent EUV source for high volume manufacturing," in Proc. 2020 EUVL Workshop, https://drive.google.com/file/d/1Ux0yPvtzG78cZ_u_ X3ZdanqY2TLqy4d9/view (2020).

20. J. Na et al., "Application of actinic mask review system for the preparation of HVM EUV lithography with defect free mask," Proc. SPIE 10145, 101450M (2017).

21. P. P. Naulleau et al., "Electrooptical system for scanning microscopy of extreme ultraviolet masks with a high harmonic generation source," Opt. Express 22 (17), 20144-20154 (2014).

22. M. Müller et al., "EUV damage threshold measurements of Mo/Si multilayer mirrors," Appl. Phys. A 108, 263 (2012).

23. J. Blanchard, "Roughening mechanisms for heated surfaces," 2002, https://blanchard.engr .wisc.edu/res/BlanchardRoughening.pdf.

24. S. Bruijn et al., "Thermal stability and lifetime scaling of multilayer EUVL optics," 2009, http://euvlsymposium.lbl.gov/pdf/2009/poster/P012_Bruijn_FOMInstitute.pdf, and references therein.

25. S. P. Hau-Riege and D. G. Stearns, "Correction of figure errors on optical surfaces by laserinduced contraction of Mo/Si multilayers," Opt. Lett. 28, 456 (2003).

26. C.-H. Ban et al., "Pattern dependent distortion and temperature variation in EUV mask," Proc. SPIE 11147, 111471R (2019).

27. M. van de Kerkhof et al., "Advanced particle contamination control in EUV scanners," Proc. SPIE 10957, 109570U (2019).

28. K. Gielissen, "The nature and characteristics of particles produced by EUV sources: exploration, prevention and mitigation," $\mathrm{PhD}$ Thesis, TU Eindhoven (2009).

29. I. V. Fomenkov et al., "EUV sources for high-volume manufacturing," in EUV Lithography, V. Bakshi, Ed., SPIE Press, Bellingham, Washington (2018).

30. H. Verbraak et al., "Angular ion emission characteristics of a laser triggered tin vacuum arc as light source for extreme ultraviolet lithography," J. Appl. Phys. 108, 093304 (2010).

31. J. Pankert et al., "EUV sources for the alpha-tools," Proc. SPIE 6151, 61510Q (2006).

32. K. Koshelev et al., "Debris-free high-brightness light source based on LPP for actinic EUV microscopy and metrology applications," Proc. SPIE 10809, 108091Q (2018). 
33. O. Khodykin, "Bright and reliable Xe-based EUV source for metrology and inspection applications," in Proc. 2015 Int. Workshop EUV and Soft X-Ray Sources, Dublin, https://www.euvlitho.com/2015/S31.pdf (2015).

Larissa Juschkin received her $\mathrm{PhD}$ in atomic and plasma physics from Ruhr-University Bochum, Germany. In 2012, she was appointed to a professorship for experimental physics of EUV at RWTH Aachen University. In 2018, she joined KLA as an EUV technologist and is currently program manager for next generation wafer inspection systems using broadband plasma sources. Her scientific interests combine plasma-based radiation sources with modern nanotechnology applications, especially in the fields of nanostructuring and high-resolution measurement technology.

Daniel Wack received his BA degree in physics from Amherst College and his PhD in applied physics from Cornell University. Currently, he is a senior director in the Reticle Inspection Division at KLA engaged in development of technology, architecture, and optics for EUV mask inspection systems. He has held multiple engineering and business leadership positions in product development at KLA and Tencor, focused on high performance, production-worthy optical systems, and generating more than 30 patents. 